\title{
Divergência genética entre acessos de batata-doce utilizando caracteres fenotípicos de raiz
}

\author{
Giovani O da Silva ${ }^{1}$; Rubens Ponijaleki ${ }^{1}$; Fabio A Suinaga ${ }^{2}$ \\ 'Embrapa SPM, C. Postal 317, 89460-000 Canoinhas-SC; giovani.olegario@embrapa.br; rubens.ponijaleki@embrapa.br; 2Embrapa \\ Hortaliças, C. Postal 218, 70351-970 Brasília-DF; fabio@enph.embrapa.br
}

\begin{abstract}
RESUMO
Estudos de quantificação da divergência genética entre acessos e caracterização dos recursos genéticos têm sido de grande importância em programas de melhoramento, auxiliando na identificação de genitores e no conhecimento do material genético disponível. Os objetivos do presente trabalho foram: (1) avaliar os acessos de batata-doce pertencentes ao banco de germoplasma da Embrapa com base em caracteres de raiz; (2) calcular a importância relativa dos caracteres morfo-agronômicos quantitativos na discriminação dos acessos e, (3) obter indicações das combinações híbridas mais promissoras para cruzamentos. Foi avaliada uma coleção de 11 clones pertencentes ao Banco Ativo de Germoplasma da Embrapa por meio de um experimento conduzido na Embrapa SPM/EN, em Canoinhas-SC. Os acessos foram cultivados em condições de campo no delineamento em blocos ao acaso com quatro repetições, onde a parcela experimental foi composta por 10 plantas. As plantas de cada parcela foram colhidas e avaliadas para 12 caracteres fenotípicos de raiz. $\mathrm{O}$ estudo indicou que os caracteres número e massa total de raízes e peso específico foram os que mais contribuíram para a divergência genética. A maioria dos acessos é bastante similar quanto aos caracteres quantitativos avaliados. Porém, há a possibilidade de ganhos com a heterose, geração de variabilidade genética e de progênies superiores cruzando-se os acessos dos diferentes grupamentos formados; pois os genótipos mais contrastantes ' 1228 ', '051-1' e ' 1270 ' foram agrupados entre os melhores para várias características como massa e número total de raízes, massa comercial de raízes; os dois primeiros apresentaram elevado peso específico e o ‘1270' apresentou coloração alaranjada intensa, indicativo de alto teor de $\beta$-caroteno.
\end{abstract}

Palavras-chave: Ipomoea batatas, banco de germoplasma, dissimilaridade, importância relativa.

\section{ABSTRACT \\ Genetic divergence among sweet potato accessions based on root traits}

The estimation of the genetic divergence among accessions of a germplasm bank, as well as their characterization are very important in breeding programs in order to identify the best parental genotypes and to provide a better understanding of the available plant genetic resources. Considering this information, the objectives of this research were: (1) evaluate the sweet potato plant accessions of Embrapa Germplasm Bank based on root traits; (2) estimate the relative importance of quantitative morphoagronomic characters in the genotype discrimination; and (3) find out promising hybrid combinations for crosses. Then, 11 sweet potato clones from Embrapa Germplasm Bank were evaluated in a trial at Embrapa SPM/EN Canoinhas, Brazil. The genotypes were cultivated in field conditions on a randomized blocks experimental design with four replications; the experimental plot was composed of 10 plants. In each experimental plot the plants were harvested and 12 characteristics of the roots were evaluated. The total number and mass of roots, and the specific gravity were the characteristics with the greatest influence on the genetic divergence. Most of the evaluated genotypes were closely related considering their quantitative characteristics. However, it is possible to obtain useful gains with heterosis and the generation of superior progenies, through crosses between genotypes from the different groups; because the most contrasting genotypes ' 1228 ', '051-1' and ' 1270 ' were grouped among the best ones for various characteristics such as mass and total number of roots, marketable root mass, the first two showed a high specific weight and '1270' intense orange color, indicative of high levels of $\beta$-carotene.

Keywords: Ipomea batatas, germplasm bank, dissimilarity, relative importance.

\section{(Recebido para publicação em 24 de junho de 2011; aceito em 4 de julho de 2012) (Received on June 24, 2011; accepted on July 4, 2012)}

\begin{abstract}
$\mathrm{A}$ batata-doce (Ipomoea batatas) é a sexta hortaliça mais plantada no Brasil, e sua importância econômica e social é resultante da sua rusticidade, ampla adaptação climática e elevada capacidade de produção de energia em curto espaço de tempo. A origem exata da batata-doce não é conhecida, mas a hipótese americana é normalmente aceita, sendo a região mais provável a faixa compreendida entre o México e o norte da América do Sul. Esta hortaliça pertence à família Convolvulaceae, sendo
\end{abstract}

I. batatas o único membro hexaplóide $(2 n=6 x=90)$. A variabilidade dentro da espécie é muito alta, provavelmente devido ao alto nível de ploidia (Ritschel et al., 2010). Atualmente observa-se considerável diversidade genética em batata-doce nas diversas regiões produtoras do Brasil, oriunda de segregação sexuada e assexuada e de introduções de plantas provenientes de outras localidades. Diante deste fato, para que esta variabilidade possa ser utilizada em programas de melhoramento envol- vendo seleção de genótipos superiores, é necessário dispor de informações a respeito do germoplasma a ser utilizado, de suas potencialidades genéticas e dos parâmetros genéticos intrínsecos às características que serão melhoradas (Oliveira et al., 2000).

Segundo Miranda et al. (1988), um dos parâmetros para a escolha de genitores a serem utilizados em programas de melhoramento é a divergência genética. Esta medida é quantificada, em geral, por estatísticas multivaria- 
das, como a distância generalizada de Mahalanobis. Assim, no melhoramento de plantas, estratégias envolvendo cruzamentos entre genótipos contrastantes, possibilitam maximizar o efeito da heterose (Cruz \& Regazzi, 1997; Moura et al., 1999), sendo importante que os genitores apresentem em sua constituição genética alelos superiores e sejam adaptados às condições locais de ambiente (Buzar et al., 2007). Estudos de divergência genética têm sido de grande importância em programas de melhoramento, gerando informações sobre parâmetros utilizados na identificação de genitores que possibilitem expressar maior efeito heterótico, além de aumentarem a probabilidade de recuperar genótipos superiores nas progênies (Cruz \& Regazzi, 1997; Buzar et al., 2007; Paixão et al., 2008).

A estimativa da divergência genética auxilia na identificação da variabilidade genética (Moura et al., 1999), informa a respeito das estratégias mais adequadas para organização do germoplasma, aumenta a eficiência da amostragem de genótipos, auxilia na definição de cruzamentos artificiais, na incorporação de genes de germoplasma exótico e até na recomendação de cultivares para determinada região, quando o objetivo é ampliar a base genética das variedades utilizadas nos cultivos comerciais (Mohammadi \& Prasanna, 2003). No entanto, esta estimativa é apenas um auxílio na predição e escolha dos genitores mais promissores, pois há necessidade de que estes apresentem bom desempenho per se para as características mais importantes, que haja complementação gênica (Oliveira et al., 2000), boa capacidade de combinação e adaptação do genitor ao clima (Silva et al., 2007).

Existem na literura muitos trabalhos com batata-doce relatando a caracterização e a dissimilaridade de acessos; no entanto, baseados na sua maioria em descritores mínimos ou moleculares e não em caracteres relacionados ao rendimento de raízes, possivelmente pela menor interferência ambiental em caracteres qualitativos (Daros et al., 2002; Ritschel \& Huamán, 2002; Veasey et al., 2007; Veasey et al., 2008). Quanto à verificação da dissimilaridade genética com vistas à obtenção de variabilidade genética para características comerciais visando o melhoramento, há poucos relatos na literatura (Fabri, 2009). Este autor ainda ressalta que o sucesso no desenvolvimento de novas combinações de caracteres em futuras cultivares de batata-doce pode estar limitado pela falta de informações sobre a diversidade do germoplasma disponível em programas de melhoramento.

Neste contexto, os objetivos do presente trabalho foram: (1) caracterizar, em relação a caracteres de raiz, acessos de batata-doce pertencentes ao banco de germoplasma da Embrapa; (2) calcular a importância relativa dos caracteres morfo-agronômicos quantitativos na discriminação dos acessos e, (3) obter indicações, a partir destes dados morfológicos, das combinações híbridas mais promissoras para cruzamentos visando ganhos com a heterose, obtenção de variabilidade genética e de progênies superiores.

\section{MATERIAL E MÉTODOS}

O experimento foi conduzido na Embrapa Produtos e Mercado, Escritório de Canoinhas-SC, onde foram avaliados 11 clones pertencentes ao Banco Ativo de Germoplasma da Embrapa Hortaliças, quais são: 1270 (proveniente de Uruguaiana-RS); 127-5, 665-B e 160-4 (origem desconhecida); 080-B (Canoinhas-SC); 345-B (Roraima); 336B (Rondônia); 051-1 (Distrito Federal); 1228 e 1219 (Centro Internacional de La Papa, Peru); e Dacosta (Rio Grande do Sul), utilizada como testemunha neste experimento. Estes acessos, praticamente desconhecidos quanto a sua caracterização fenotípica, foram cultivados em condições de campo no período compreendido entre 23/12/2009 a 29/06/2010, no delineamento em blocos ao acaso com quatro repetições e parcelas formadas por 10 plantas com espaçamento de $75 \mathrm{~cm}$ entre linhas (duas linhas por parcela) e $30 \mathrm{~cm}$ entre plantas. A adubação em pré-plantio foi realizada com superfosfato triplo (261 $\mathrm{kg} \mathrm{ha}^{-1}$ ) e $\mathrm{KCl}\left(200 \mathrm{~kg} \mathrm{ha}^{-1}\right)$, as condições climáticas apresentaram-se normais para o período. Foi realizada capina/amontoa em 25/01/2010. Em 29/06/2010 foram colhidas e avaliadas as plantas de cada parcela para os caracteres número total de raízes (NTR); número de raízes com padrão comercial (NRC); número de raízes com padrão não comercial (NRNC); com utilização de balança foram avaliados: massa total de raízes (MTR), ou seja, tanto das raízes comerciais como não comerciais; massa fresca de raízes com padrão comercial (MRC); massa fresca de raízes com padrão não comercial (MRNC); avaliou-se também o peso específico das raízes comerciais (PE) pela fórmula: [peso no ar / (peso no ar / peso na água)], com auxílio de um dinamômetro; o comprimento de raízes $(\mathrm{cm})$ (COMP), com utilização de régua plástica graduada; e o diâmetro das raízes (DIAM), com utilização de paquímetro; por observação visual, e sem a utilização de uma escala de cores, foram avaliadas a coloração externa da raiz (Cor-E); coloração interna da raiz (Cor-I); coloração interna da raiz após o cozimento por 30 minutos (Cor-AC). Foram consideradas como raízes comerciais aquelas acima de $10 \mathrm{~cm}$ de comprimento e $5 \mathrm{~cm}$ de diâmetro, além de não tortas, embonecadas ou rachadas.

Os dados obtidos foram submetidos à análise de variância e agrupamento de médias por Scott \& Knott a 5\%, com exceção para os caracteres de coloração. Posteriormente, com a utilização dos caracteres quantitativos foram efetuadas análises de dissimilaridade entre os acessos pela divergência generalizada de Mahalanobis e da importância relativa dos caracteres, estimada por meio da participação dos componentes de $\mathrm{D}^{2}$, relativos a cada caractere, no total da dissimilaridade observada (Singh, 1981). Todas estas estimativas foram realizadas através do programa GENES (Cruz, 2001). As matrizes de dissimilaridade foram transferidas para o programa NTSYSpc (Rohlf, 2000), para obtenção do agrupamento em dendrograma pelo método de agrupamento das divergências médias (UPGMA) e diagnóstico das correlações cofenéticas entre as matrizes e os agrupamentos (Rohlf \& Sokal, 1981).

\section{RESULTADOS E DISCUSSÃO}

Todos os valores obtidos para o quadradro médio de tratamentos para 
Tabela 1. Contribuição relativa de nove caracteres fenotípicos de raizes de batatadoce para a divergência (Singh, 1981), em porcentagem (relative contribution of nine traits of sweet potato roots to the divergence (Singh, 1981), in percentage). Canoinhas, Embrapa SPM, 2010.

\begin{tabular}{lc}
\hline Caracteres & $\begin{array}{c}\text { Contribuição } \\
\text { relativa }(\%)\end{array}$ \\
\hline NTR & 32,84 \\
NRC & 16,80 \\
NRNC & 5,55 \\
MTR & 16,00 \\
MRC & 1,99 \\
MRNC & 4,27 \\
PE & 18,62 \\
COMP & 3,18 \\
DIAM & 0,71 \\
\hline
\end{tabular}

$\mathrm{NTR}=$ número total de raízes; $\mathrm{NRC}=$ número de raízes com padrão comercial; $\mathrm{NRNC}=$ número de raízes com padrão não comercial; $\mathrm{MTR}=$ massa total de raízes; $\mathrm{MRC}=$ massa de raízes com padrão comercial; $\mathrm{MRNC}=$ massa de raízes com padrão não comercial; $\mathrm{PE}=$ peso específico $\mathrm{COMP}=$ comprimento de raízes; $\mathrm{DIAM}=$ diâmetro de raízes $(\mathrm{NTR}=$ total number of roots; $\mathrm{NRC}=$ number of roots with market standard; $\mathrm{NRNC}=$ number of roots with no market standard; $\mathrm{MTR}=$ total mass of roots; $\mathrm{MRC}=$ root mass with market standard; $\mathrm{MRNC}=$ root mass with no market standard; $\mathrm{PE}=$ specific gravity; $\mathrm{COMP}=$ root length; DIAM= root diameter; Cor-E= external color of the root; Cor-I= internal color of the root; Cor-AC $=$ root internal color after cooking).

os caracteres em estudo foram significativos, possibilitando diferenciar os acessos. Os coeficientes de variação fenotípica apresentaram desde valores baixos como para peso específico $(1,78 \%)$ até valores elevados e superiores a $25 \%$ para NRC, NRNC, MTR, MRC e MRNC, que demonstraram ser altamente influenciados pelo ambiente (Tabela 1), conforme confirmado pela literatura (Azevedo et al., 2000; Cardoso et al., 2005; Queiroga et al., 2007; Andrade Junior et al., 2009).

Extrapolando o valor médio do caráter massa total de raízes por parcela para um hectare obteve-se $33,4 \mathrm{t} \mathrm{ha}^{-1}$, rendimento esse semelhante aos obtidos por Azevedo et al. (2000) e Andrade Junior et al. (2009). Em comparação com o rendimento médio brasileiro que

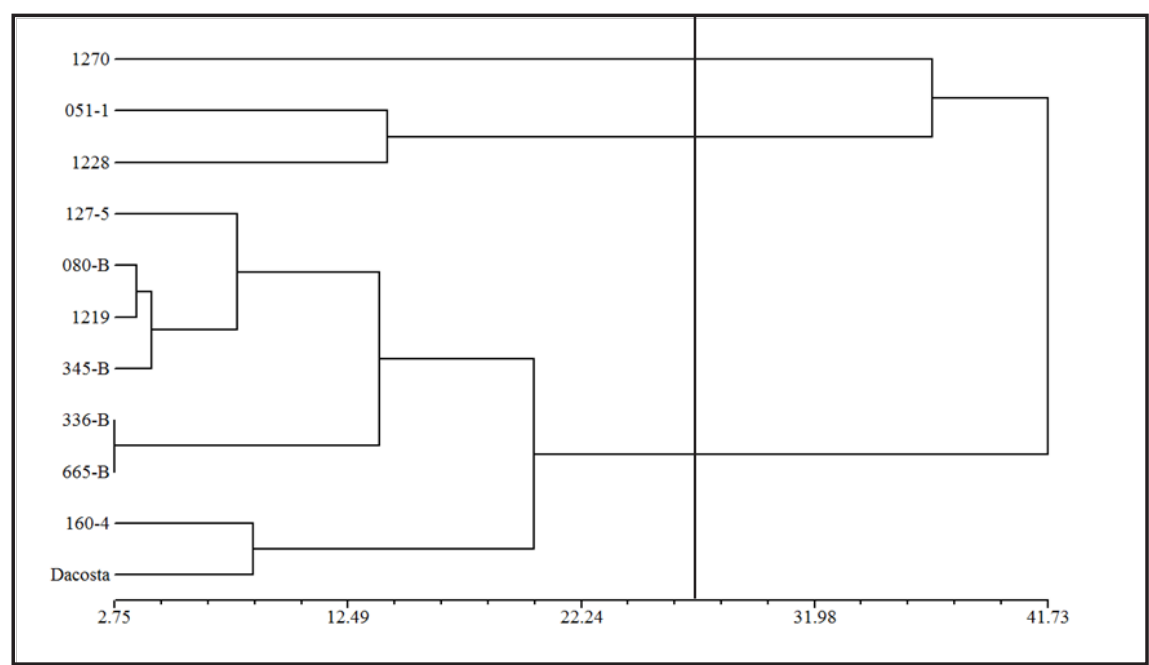

Figura 1. Distâncias morfológicas de Mahalanobis entre 11 acessos de batata doce, obtidas a partir da avaliação de nove caracteres de raiz; coeficiente de determinação 0,73 , distância média 27,09 (Mahalanobis morphological distances between 11 accessions of sweet potato obtained from the evaluation of nine root traits, coefficient of determination 0.73 , mean distance 27.09). Canoinhas, Embrapa SPM, 2010.

é de 11,80 tha-1 segundo o Instituto Brasileiro de Geografia e Estatística em 2010 (IBGE, 2010), pode-se verificar que os acessos possuem um potencial produtivo superior, porém, sabe-se que o rendimento brasileiro, além do potencial produtivo dos genótipos depende de melhorias na sanidade e manejo.

Quanto à importância relativa dos caracteres estudados na dissimilaridade entre os acessos, verifica-se que o número total de raízes, a massa total de raízes e o peso específico apresentaram maior porcentagem de contribuição, ou seja, os agrupamentos dos acessos foram predominantemente influenciados por essas três características; isso permite concluir também que os acessos diferem mais uns dos outros para estas características do que para as demais. Já o diâmetro e a massa de raízes comerciais foram bastante similares entre os acessos (Tabela 1).

O dendrograma representativo da divergência entre os genótipos pode ser visualisado na Figura 1. O coeficiente de correlação cofenética, que estima a representatividade dos dados da matriz de dissimilaridade no dendrograma, foi de 0,73 , indicando que os dados da matriz estão bem ajustados no dendrograma (Mantel, 1967).

De acordo com a divergência média entre os acessos $(27,09)$, houve a formação de três agrupamentos, sendo um grupo maior composto por oito dos 11 acessos avaliados, e dois grupamentos menores, um composto por dois acessos '1228' e '051-1' e outro formado por apenas um acesso ' 1270 '. Esta distribuição indica que em relação aos caracteres avaliados, a maioria dos acessos apresentou níveis elevados de similaridade. Assim, para maximizar a heterose, os componentes dentro de cada grupo não deveriam ser cruzados entre si (Luthra et al., 2005).

Dentro do grupo maior, os acessos '336-B' e 665-B' apresentaram similaridade elevada, apesar de não serem duplicatas, conforme verificado pelas avaliações de coloração. Cruzamentos entre estes acessos provavelmente não seriam efetivos na obtenção de variabilidade genética para os caracteres quantitativos avaliados.

De acordo com a análise de agrupamento de médias por Scott \& Knott (Tabela 2), os acessos com maior número de raízes com características comerciais foram '127-5', '1228', '1270', '080B, '051-1 e '1219'. Considerando os caracteres número de raízes comerciais e não comerciais, o '127-5' e '1228' apresentaram o maior número total de raízes; e também, juntamente com a cultivar testemunha Dacosta, apresentaram o maior número de raízes com características não comerciais (Tabela 2).

Com exceção dos acessos '336-B', 
Tabela 2. Comparação de médias por Scott-Knott para caracteres fenotípicos avaliados em batata-doce (comparison of means by Scott-Knott for traits evaluated in sweet potato). Canoinhas, Embrapa SPM, 2010.

\begin{tabular}{lcccccc}
\hline Acessos & NTR & NRC & NRNC & MTR(g) & MRC(g) & MRNC(g) \\
\hline 1270 & $51,00 \mathrm{~b}$ & $30,75 \mathrm{a}$ & $20,50 \mathrm{~b}$ & $12858,50 \mathrm{a}$ & $6837,50 \mathrm{a}$ & $6021,00 \mathrm{a}$ \\
$127-5$ & $61,75 \mathrm{a}$ & $28,50 \mathrm{a}$ & $33,00 \mathrm{a}$ & $9522,25 \mathrm{a}$ & $7481,00 \mathrm{a}$ & $2041,50 \mathrm{~b}$ \\
$080-\mathrm{B}$ & $44,75 \mathrm{~b}$ & $25,50 \mathrm{a}$ & $19,25 \mathrm{~b}$ & $9951,25 \mathrm{a}$ & $8348,00 \mathrm{a}$ & $1603,25 \mathrm{~b}$ \\
$345-\mathrm{B}$ & $42,75 \mathrm{~b}$ & $21,75 \mathrm{~b}$ & $21,00 \mathrm{~b}$ & $6567,00 \mathrm{~b}$ & $5473,25 \mathrm{a}$ & $1093,75 \mathrm{~b}$ \\
$336-\mathrm{B}$ & $32,25 \mathrm{c}$ & $18,25 \mathrm{~b}$ & $14,00 \mathrm{~b}$ & $4157,75 \mathrm{~b}$ & $3255,00 \mathrm{~b}$ & $902,50 \mathrm{~b}$ \\
$051-1$ & $40,75 \mathrm{~b}$ & $28,75 \mathrm{a}$ & $12,25 \mathrm{~b}$ & $8884,75 \mathrm{a}$ & $6755,25 \mathrm{a}$ & $2129,50 \mathrm{~b}$ \\
1228 & $57,75 \mathrm{a}$ & $30,25 \mathrm{a}$ & $27,25 \mathrm{a}$ & $8458,00 \mathrm{a}$ & $6556,25 \mathrm{a}$ & $1901,75 \mathrm{~b}$ \\
$665-\mathrm{B}$ & $32,00 \mathrm{c}$ & $19,50 \mathrm{~b}$ & $12,50 \mathrm{~b}$ & $5175,25 \mathrm{~b}$ & $3571,25 \mathrm{~b}$ & $1604,00 \mathrm{~b}$ \\
1219 & $42,00 \mathrm{~b}$ & $24,75 \mathrm{a}$ & $17,25 \mathrm{~b}$ & $7280,25 \mathrm{~b}$ & $5679,75 \mathrm{a}$ & $1600,50 \mathrm{~b}$ \\
$160-4$ & $24,75 \mathrm{c}$ & $15,00 \mathrm{~b}$ & $10,00 \mathrm{~b}$ & $4709,50 \mathrm{~b}$ & $3249,00 \mathrm{~b}$ & $1461,00 \mathrm{~b}$ \\
Dacosta & $46,50 \mathrm{~b}$ & $18,00 \mathrm{~b}$ & $28,50 \mathrm{a}$ & $5106,25 \mathrm{~b}$ & $3358,00 \mathrm{~b}$ & $1748,50 \mathrm{~b}$ \\
\hline Média & 43,30 & 23,73 & 19,59 & 7515,52 & 5505,84 & 2009,75 \\
CV & 19,97 & 25,26 & 36,25 & 32,47 & 35,95 & 54,37 \\
\hline Acessos & PE & COMP(cm) & DIAM(cm) & Cor-E & Cor-I & Cor-AC \\
\hline 1270 & $1,093 \mathrm{~b}$ & $10,77 \mathrm{~b}$ & $9,77 \mathrm{a}$ & Rosada & Laranja & Laranja \\
$127-5$ & $1,079 \mathrm{c}$ & $13,42 \mathrm{a}$ & $7,17 \mathrm{~b}$ & Rosada & Branca & Amarelado \\
$080-\mathrm{B}$ & $1,064 \mathrm{c}$ & $14,35 \mathrm{a}$ & $6,70 \mathrm{~b}$ & Salmão & Branca & Creme \\
$345-\mathrm{B}$ & $1,079 \mathrm{c}$ & $12,17 \mathrm{~b}$ & $5,62 \mathrm{~b}$ & Branca & Branca & Creme \\
$336-\mathrm{B}$ & $1,094 \mathrm{~b}$ & $12,10 \mathrm{~b}$ & $6,65 \mathrm{~b}$ & Rosada & Branca & Laranja \\
$051-1$ & $1,135 \mathrm{a}$ & $10,85 \mathrm{~b}$ & $8,70 \mathrm{a}$ & Branca & Branca & Caramelo \\
1228 & $1,147 \mathrm{a}$ & $12,92 \mathrm{a}$ & $7,32 \mathrm{~b}$ & Branca & Branca & Creme \\
$665-\mathrm{B}$ & $1,113 \mathrm{~b}$ & $11,42 \mathrm{~b}$ & $5,97 \mathrm{~b}$ & Caramelo & Laranja & Creme \\
1219 & $1,049 \mathrm{~d}$ & $13,55 \mathrm{a}$ & $8,32 \mathrm{a}$ & Roxa & Branca & Creme \\
$160-4$ & $1,040 \mathrm{~d}$ & $14,92 \mathrm{a}$ & $6,87 \mathrm{~b}$ & Rosa & Branca & Creme \\
Dacosta & $1,045 \mathrm{~d}$ & $13,42 \mathrm{a}$ & $6,25 \mathrm{~b}$ & Creme & Creme & Creme \\
\hline Média & 1,09 & 12,72 & 7,22 & - & - & - \\
CV & 1,78 & 13,50 & 23,67 & - & - & - \\
\hline
\end{tabular}

$\mathrm{NTR}=$ número total de raízes; $\mathrm{NRC}=$ número de raízes com padrão comercial; $\mathrm{NRNC}=$ número de raízes com padrão não comercial; $\mathrm{MTR}=$ massa total de raízes; $\mathrm{MRC}=$ massa de raízes com padrão comercial; $\mathrm{MRNC}=$ massa de raízes com padrão não comercial; $\mathrm{PE}=$ peso específico; $\mathrm{COMP}=$ comprimento de raízes; $\mathrm{DIAM}=$ diâmetro de raízes; Coe-E= coloração externa da raiz; Cor-I= coloração interna da raiz; Cor- $\mathrm{AC}=$ coloração interna da raiz após o cozimento $(\mathrm{NTR}=$ total number of roots; $\mathrm{NRC}=$ number of roots with market standard; $\mathrm{NRNC}=$ number of roots with no market standard; $\mathrm{MTR}=$ total mass of roots; $\mathrm{MRC}=$ root mass with market standard; $\mathrm{MRNC}=$ root mass with no market standard; $\mathrm{PE}=$ specific gravity; $\mathrm{COMP}=$ root length; $\mathrm{DIAM}=$ root diameter; $\mathrm{Cor}-\mathrm{E}=$ external color of the root= $\mathrm{Cor}-\mathrm{I}=\mathrm{internal}$ color of the root; Cor- $\mathrm{AC}=$ root internal color after cooking).

'665-B' e '160-4' e da cultivar testemunha Dacosta, os demais genótipos mostraram-se superiores em relação à massa fresca de raízes comerciais. O acesso ' 1270 ' apresentou a maior massa fresca de raízes não comerciais, indicando que este teve muitas raízes desclassificadas por problemas de deformações. Desconsiderando os genótipos '345-B' e '1219', os acessos com maior massa fresca total de raízes também foram os que apresentaram as maiores massas fresca de raízes comerciais e maior número de raízes comerciais (Ta- bela 2). Este tipo de análise comprova a importância da classificação das raízes nas diferentes classes, comerciais e não comerciais no momento das avaliações; pois, por exemplo, pela massa total de raízes (MTR) os acessos '345-B' e ' 1219 ' não foram classificados como mais produtivos, porém considerando apenas as raízes comerciais estiveram agrupados com aqueles com maiores valores. Por outro lado, a obtenção de maior produção total aliada à maior produção comercial é interessante para os casos onde os produtores possuem outras formas de utilização das raízes não comerciais, como por exemplo, a alimentação animal, consumo próprio, mercados menos exigentes ou produção de doces e farinhas.

Quanto ao peso específico, parâmetro relacionado ao teor de matéria seca, os acessos com os maiores valores foram '051-1'e '1228' (Tabela 2). O teor de matéria seca influencia na crocância quando o material é utilizado para fritura e maior rendimento quando utilizado na produção de farinha (Leonel \& Cereda, 2002). 
Pode-se verificar que, em geral, os acessos com raízes mais compridas apresentaram menores diâmetros de raiz. O único acesso que apresentou os maiores diâmetro e comprimento de raízes foi o ' 1219 '. Além disto, foi possível verificar grande variabilidade para coloração externa de raiz. Já para a coloração interna, a maioria dos genótipos apresentou cor branca, e para a coloração da polpa após o cozimento, prevaleceu a cor creme na maioria dos acessos (Tabela 2). A preferência pelas características de cor, tanto interna quanto externa, depende dos consumidores e não há unanimidade (Nobrega, 2011). No entanto, sabe-se que a batata-doce com polpa alaranjada contém maior quantidade de beta-caroteno (Miranda et al., 1988), e neste quesito, os acessos '665-B' e '1270' apresentaram polpa alaranjada antes do cozimento e o ‘1270' manteve esta coloração mesmo após o cozimento.

Em síntese, o presente estudo indicou que os caracteres número e massa total de raizes e peso específico foram os que mais contribuíram para a divergência genética. A maioria dos acessos é bastante similar quanto aos caracteres quantitativos avaliados. Porém, há a possibilidade de ganhos com a heterose, geração de variabilidade genética e de progênies superiores cruzando-se os acessos dos diferentes grupamentos formados, pois os genótipos mais contrastantes '1228', '051-1'e '1270' foram agrupados entre os melhores para várias características como massa e número total de raízes, massa comercial de raízes; os dois primeiros apresentaram elevado peso específico e o último, coloração alaranjada intensa, indicativo de alto teor de beta-caroteno.

\section{REFERÊNCIAS}

ANDRADE JUNIOR VC; VIANA DJS; FERNANDES JSC; FIGUEIREDO JA; NUNES UR; NEIVA IP. 2009. Selection of sweet potato clones for the region Alto Vale do Jequitinhonha. Horticultura Brasileira 27: 389-393.

AZEVEDO SM; FREITAS JA; MALUF WR;
SILVEIRA MA. 2000. Desempenho de clones e métodos de plantio de batata-doce. Acta Scientiarum 22: 901-905.

BUZAR AGR; OLIVEIRA VR; BOITEUX LS. 2007. Estimativa da diversidade genética de germoplasma de cebola via descritores morfológicos, agronômicos e bioquímicos. Horticultura Brasileira 25: 513-518.

CARDOSO AD; VIANA AES; RAMOS PAS; MATSUMOTO SN; AMARAL CLF; SEDIYAMA T; MORAIS OM. 2005. Avaliação de clones de batata-doce em Vitória da Conquista. Horticultura Brasileira 23: 911-914.

CRUZ CD; REGAZZI AJ. 1997. Divergência genética In: CRUZ CD; REGAZZI AJ. Métodos Biométricos Aplicados ao Melhoramento Genético. Viçosa, UFV: Imprensa Universitária 6: 287-324.

CRUZ CD. 2001. Programa Genes: Aplicativo computacional em genética e estatística. Viçosa, Editora UFV, 648p.

DAROS M; AMARAL JÚNIOR AT; PEREIRA TNS; LEAL NR; FREITAS SP; SEDIYAMA T. 2002. Caracterização morfológica de acessos de batata-doce. Horticultura Brasileira 20: 43-47.

FABRI EG. 2009. Diversidade genética entre acessos de batata-doce (Ipomoea batatas L. Lam.) avaliados através de marcadores microssatélites e descritores morfoagronômicos. Piracicaba: Escola Superior de Agronomia Luiz de Queiroz. 60p. (Tese doutorado).

IBGE - INSTITUTO BRASILEIRO DE GEOGRAFIA E ESTATÍSTICA. 2010. Produção Agrícola Municipal 2010: informações sobre culturas temporárias. Rio de Janeiro: IBGE. Disponível em: http://www. sidra.ibge.gov.br/bda/pesquisas/pam/default. asp. Acessado em 02 de fevereiro de 2012.

LEONEL M; CEREDA MP. 2002. Caracterização físico-química de algumas tuberosas amiláceas. Ciência e Tecnologia de Alimentos 22: 65-69.

LUTHRA SK; GOPAL J; SHARNA PC. 2005. Genetic divergence and its relationship with heterosis in potato. Indian Potato Journal 32: $37-42$.

MANTEL N. 1967. The detection of disease clustering and a generalized regression approach. Cancer Research 27: 209-220.

MIRANDA JEC; CRUZ CD; PEREIRA AS. 1988. Análise de trilha e divergência genética de cultivares e clones de batata-doce. Revista Brasileira de Genética 11: 881-892.

MOHAMMADI SA; PRASANNA BM. 2003. Analyses of genetic diversity in crop plants - Salient statistics tools and considerations. Crop Science 43: 1235-1248.

MOURA WM; CASALI VWD; CRUZ CD; LIMA PC. 1999. Divergência genética em linhagens de pimentão em relação à eficiência nutricional de fósforo. Pesquisa Agropecuária Brasileira 34: $217-224$.
NOBREGA DS. 2011. Reação de clones de batatadoce aos nematóides de galhas do gênero Meloidogyne sp. Brasilia: Universidade de Brasília, Faculdade de Agronomia e Medicina Veterinária. 43p. (Monografia).

OLIVEIRA ACB; SEDIYAMA MAN; SEDIYAMA T; CRUZ CD. 2000. Avaliação da divergência genética em batata-doce por procedimentos multivariados. Acta Scientiarum 22: 895-900.

PAIXÃO SL; CAVALCANTE M; FERREIRA PV; MADALENA JAS; PEREIRA RG. 2008. Divergência genética e avaliação de populações de milho em diferentes ambientes no estado de Alagoas. Caatinga 21: 191-195.

QUEIROGA RCF; SANTOS MA; MENEZES MA; VIEIRA CPG; SILVA MC. 2007. Fisiologia e produção de cultivares de batata-doce em função da época de colheita. Horticultura Brasileira 25: 371-374.

RITSCHEL PS; HUAMÁN Z. 2002. Variabilidade morfológica da coleção de germoplasma de batata-doce da Embrapa - Centro Nacional de Pesquisa de Hortaliças. Pesquisa Agropecuária Brasileira 37: 485-492.

RITSCHEL PS; LOPES CA; HUAMÁN Z; FERREIRAME; FRANCAFJ; MENÊZES JE; TEIXEIRADMC; TORRESAC; CHARCHAR JM; THOMAZELLI L. 2010. Organização do banco ativo de germoplasma de batata-doce: situação atual e perspectivas. In: QUEIROZ MA; GOEDERT CO; RAMOS SRR. (eds). Recursos genéticos e melhoramento de plantas para o Nordeste brasileiro: versão 1.0. Disponível em: <http:www.cpatsa.embrapa. br>. Acesso em: 26 de agosto.

ROHLF FJ. 2000. NTSYSpc Numerical Taxonomy and Multivariate Analysis System Version 2.1. Exeter Software, Setauket, NY.

ROHLF FJ; SOKAL RRN. 1981. Comparing numerical taxonomic studies. Systematic Zoology 30: 459-499.

SILVA GO; PEREIRA AS; SOUZA VQ; CARVALHO FIF; NEY VG. 2007. Influência da distância entre genitores de batata nas estimativas de capacidades de combinação em gerações iniciais de seleção. Magistra 19: $177-264$.

SINGH D. 1981. The relative importance of characters affecting genetic divergence. The Indian Journal of Genetics and Plant Breeding 41: 237-245.

VEASEY EA; SILVA JRQ; ROSA MS; BORGES A; BRESSAN EA; PERONI N. 2007. Phenology and morphological diversity of sweet potato (Ipomoea batatas) landraces of the Vale do Ribeira. Science Agriculture 64: 416-427.

VEASEY EA; BORGES A; ROSA MS; QUEIROZ-SILVA JR; BRESSAN EA; PERONI N. 2008. Genetic diversity assessed with microsatellites in Brazilian sweetpotato (Ipomoea batatas (L.) Lam) landraces. Genetical and Molecular Biology 31: 725-733. 